\title{
Seventeen years of life support courses for nurses: where are we now?
}

Kenneth $\underline{\text { Heng }}^{1}$, MBBS, FRCS, Fong Chi Wee ${ }^{2}$, MSH, MEd

\begin{abstract}
The Life Support Course for Nurses (LSCN) equips nurses with the resuscitation skills to be first responders in in-hospital cardiac arrests. Seventeen years after the initiation of the LSCN, a confidential cross-sectional Qualtrics ${ }^{\mathrm{TM}}$ survey was conducted in May 2016 on LSCN graduands to assess the following: confidence in nurse-initiated resuscitation post-LSCN; defibrillation experience and outcomes; and perceived barriers and usefulness of the LSCN. The majority of respondents reported that the course was useful and enhanced their confidence in resuscitation. Skills retention can be enhanced by organising frequent team-based resuscitation training. Resuscitation successes should be publicised to help overcome perceived barriers.
\end{abstract}

Keywords: in-hospital cardiac arrest, life support course for nurses, nurse-initiated resuscitation

\section{INTRODUCTION}

The in-hospital cardiac arrest (IHCA) chain of survival includes the following: (1) surveillance and prevention; (2) recognition and activation of hospital cardiac arrest (Code Blue) teams; (3) uninterrupted high-quality cardiopulmonary resuscitation (CPR); (4) rapid defibrillation; and (5) advanced cardiac life support and post-resuscitation care. ${ }^{(1)}$ It has been reported that only $10 \%-20 \%$ of IHCA patients survive till discharge. ${ }^{(2-5)}$ In contrast to out-of-hospital cardiac arrest (OHCA) rhythms, the initial IHCA rhythm is usually non-shockable. ${ }^{(4-5)}$ This is possibly due to the higher incidence of physiological deterioration with hypoxaemia and hypotension, rather than the acute coronary event. The key to prevention of IHCA is surveillance, through the use of early warning scores, ${ }^{(6)}$ and escalation of care to medical emergency teams. ${ }^{(7-8)}$ Donnino et al reported that for IHCA with non-shockable rhythms, early administration of adrenaline within 1-3 minutes, compared with longer administration times, was associated with increased return of spontaneous circulation (ROSC), survival to hospital discharge and neurologically intact survival. ${ }^{(9)}$ As the usual first responders in IHCA, nurses thus play an important role, whether in administering adrenaline, performing CPR or defibrillation.

While the Basic Cardiac Life Support (BCLS) course is mandatory for all nurses in Singapore, not many nurses deployed outside of critical care areas attend the Advanced Cardiac Life Support (ACLS) course. The Life Support Course for Nurses (LSCN) was developed in $1999^{(10)}$ under the auspices of the National Resuscitation Council (NRC), Singapore, to bridge the gap between BCLS and ACLS. The LSCN teaches advanced airway skills such as laryngeal mask insertion; endotracheal intubation; resuscitation algorithms for ventricular fibrillation or pulseless ventricular tachycardia and asystole/pulseless electrical activity; defibrillation with automated and manual defibrillators; and simplified drug therapy. The course enables nurses to institute effective advanced care for IHCA (steps 2-4 in the chain of survival) before the arrival of Code Blue teams. Recognising that the work environment of Singapore nurses varies widely and nursing governance is not homogeneous, a general framework has been provided so that local practice can be adapted during nurseinitiated resuscitation. The 2016 cardiac arrest LSCN algorithm (Fig. 1) reflects the key emphasis of the NRC's 2016 guidelines: good quality, uninterrupted CPR delinked from ventilation; and early defibrillation.

\section{QUALTRICS SURVEY ON LSCN}

In Singapore, there are currently ten LSCN provider training centres and three LSCN instructor training centres with 232 active instructors. To date, 2,211 new certification courses and 1,149 recertification courses have been conducted. Over a decade after the implementation of the LSCN, we sought to take stock by conducting a confidential cross-sectional Qualtrics ${ }^{\mathrm{TM}}$ survey in May 2016. The survey consisted of five parts: (1) demographics of the respondent; (2) confidence in nurse-initiated resuscitation pre- and post-LSCN; (3) defibrillation experience and outcomes post-LSCN; (4) perceived barriers to nurse-initiated resuscitation; and (5) perceived usefulness of LSCN and reasons for perceptions.

\section{RESULTS}

There were 305 respondents to the survey (Table I). Mirroring the gender bias of nurses in Singapore, $92 \%$ of respondents were female. The mean age of the respondents was 38 (range $23-72$; median 40 ) years. The majority (66\%) had more than ten years of nursing experience (Table I). $64 \%$ of the respondents were registered nurses, $10 \%$ were nurse managers and $26 \%$ were LSCN instructors. The reported confidence in performing defibrillation was statistically significantly higher post-LSCN ( $p<0.001$, two-tailed Fisher's exact test). Following the LSCN, $39 \%$ of respondents had performed defibrillation, while $61 \%$ had not. The survey showed that $84 \%$ of nurse-initiated resuscitations achieved ROSC.

Among the respondents, $70 \%$ opined that the LSCN was very useful, while $27 \%$ reported moderate usefulness. The remaining 


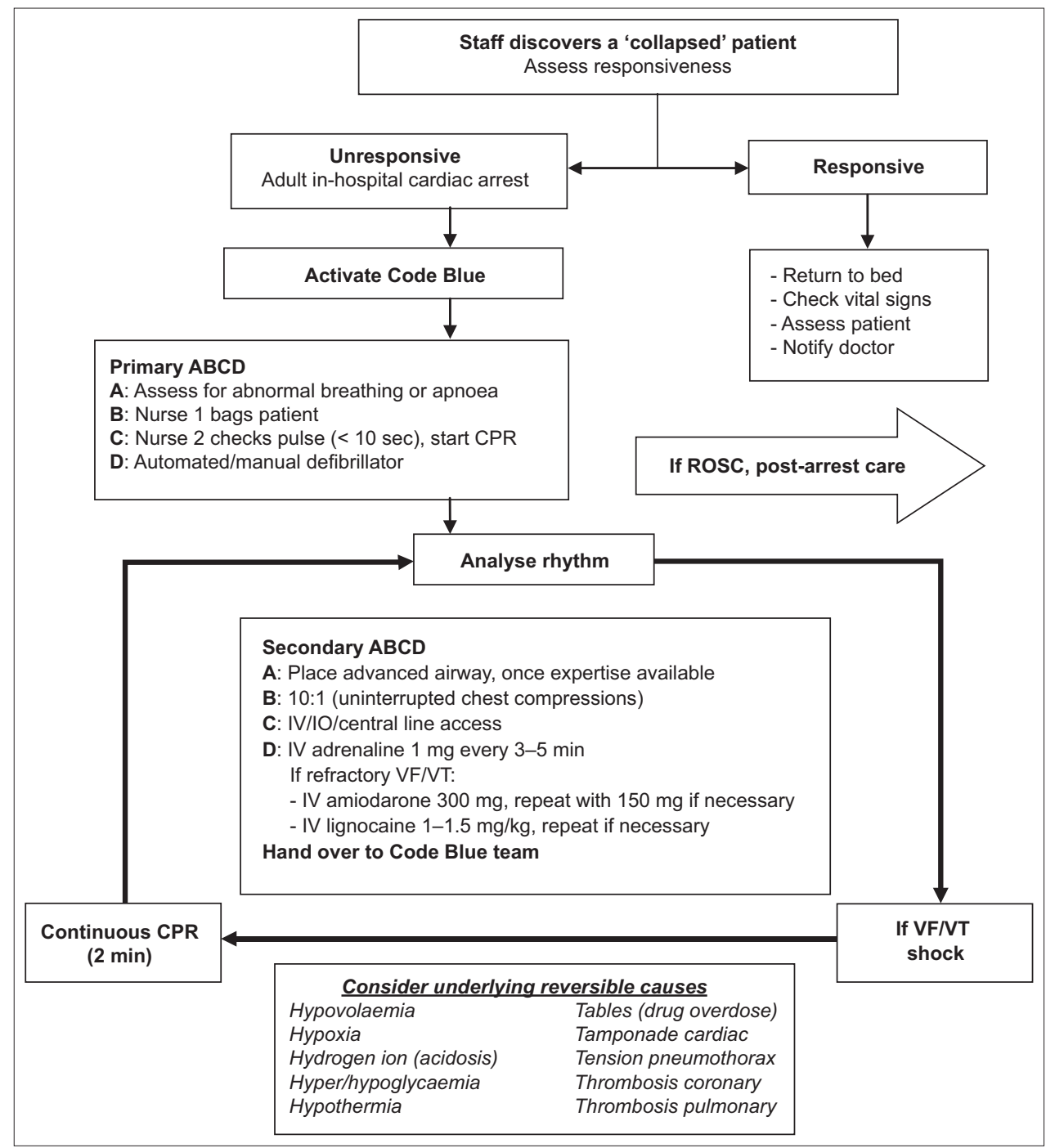

Fig. 1 Life Support Course for Nurses cardiac arrest algorithm. CPR: cardiopulmonary resuscitation; IO: intraosseous infusion; IV: intravenous; ROSC: return of spontaneous circulation; VF: ventricular fibrillation; VT: ventricular tachycardia

Table I. Demographics of survey respondents $(n=305)$.

\begin{tabular}{lc}
\hline Characteristic & No. (\%) \\
\hline Gender & \\
$\quad$ Female & $280(92)$ \\
Work experience (yr) & \\
$<2$ & $4(1)$ \\
$2-5$ & $37(12)$ \\
$5-10$ & $62(20)$ \\
$>10$ & $202(66)$ \\
Role (n = 301) & $190(63)$ \\
Nurse & $32(11)$ \\
Manager & $79(26)$ \\
LSCN trainer
\end{tabular}

LSCN: Life Support Course for Nurses

$3 \%(n=9)$ of respondents found the course not useful: six attributed it to the lack of opportunity to use the skills learnt (due to where they were deployed), which led to degradation of skills; two cited that certain components of the course (like endotracheal intubation) should not have been taught, as they were not accredited to perform such procedures; and one mentioned trainer competence issues. The main barriers to nurse-initiated resuscitation were: lack of confidence (35\%); fear of litigation $(13 \%)$; fear of harming the patient $(22 \%)$; hospital policy $(21 \%)$; and lack of opportunity (10\%) due to various reasons (Table II).

\section{DISCUSSION}

It would appear from the survey results that graduates of the LSCN tend to be more senior. To maximise the number of clinical areas staffed with LSCN-trained nurses, it is recommended that the LSCN feature earlier in nurses' continuing education roadmap. The majority of LSCN graduates $(61 \%)$ stated that they had not defibrillated. This is likely a reflection of where they were deployed; for example, in subacute wards or clinics, IHCAs do not commonly occur.

\section{Barriers to nurse-initiated resuscitation}

Young et al reported insufficient training (37.7\%), lack of competence $(9.8 \%)$, lack of self-confidence $(5.9 \%)$, and workload and tension $(5.1 \%)$ as the leading barriers to nurse-initiated resuscitation. ${ }^{(11)}$ To mitigate these barriers, it was suggested that "strategic planning and resuscitation skills training should be 
Table II. Barriers faced in nurse-initiated resuscitation.

\begin{tabular}{ll}
\hline Barrier & No. (\%) \\
\hline Lack of confidence & $54(35)$ \\
Fear of litigation & $20(13)$ \\
Fear of harming patient & $34(22)$ \\
Hospital policy does not allow & $32(21)$ \\
No opportunity* & $15(10)$
\end{tabular}

155 respondents identified barriers faced in nurse-led resuscitation. * Reasons include: work in a teaching institution $(n=3)$; doctor is always around $(n=5)$; have not encountered a cardiac arrest yet $(n=5)$; others $(n=2)$.

incorporated into staff development programmes to reduce barriers to optimal resuscitation performance and cope with work demands for ward nurses". Locally, it has been previously postulated that the lack of confidence, fear of incurring litigation, fear of harming the patient, lack of practising privilege by the nursing governance, entrenched 'supportive' role of nurses in resuscitation and perceived difficulty in interpreting electrocardiograph rhythms were barriers to nurse-initiated resuscitation. ${ }^{(12)}$

In this survey, approximately half the respondents perceived that the first four barriers still exist. Given the encouraging rates of reported ROSC after nurse-initiated defibrillation, publicity of successful nurse-initiated resuscitations by nurse leaders may gradually improve these perceptions. With the option of using the automated external defibrillator function, the barrier of difficulty in interpreting electrocardiograph rhythms no longer exists.

\section{Retention of resuscitation skills in nurses}

Some survey respondents raised the issue of skills retention and degradation. The principles of the LSCN adhere to evidencebased education concepts, including blended learning pedagogy involving computer-based learning for core content and handson practice for skills learning to aid retention. Contextualised resuscitation training is also conducted to test application, analysis and evaluation (higher rungs of Bloom's Taxonomy ${ }^{(13)}$ ). Structured feedback and debriefing sessions are carried out by instructors and clear remediation goals are set for improvement. In addition, participants are encouraged to undertake reflective learning at the end of each session. In June 2014, a faculty development session on LSCN multiple-choice standard setting was organised for LSCN instructor leads.

It is well known that CPR skills degrade more rapidly than knowledge. Skills appear to decline as early as two weeks after training, diminishing to pre-training levels by $1-2$ years. ${ }^{(14)}$ Leith noted that a sample of intensive care nurses retained knowledge over a six- and 12-month period, but were unable to achieve the standard required to pass the practical test in that time. ${ }^{(15)}$ In a review article, Hamilton reported different methods for improving skills retention: simulation training; peer tuition; video self-instruction; computer-based learning programmes; voice-activated manikins; automated external defibrillators; selfinstruction; gaming; and the use of action cards. ${ }^{(16)}$

Significantly more respondents in the study reported improved confidence in nurse-initiated resuscitation after the LSCN. However, the majority of respondents who opined that the course was not useful experienced a lack of opportunity to use the skills learnt, resulting in degradation of skills. Similarly, Murphy reported that although many nurses felt confident immediately after a life support course, most felt that as time passed, their confidence had reduced to such a degree that they would not use their skills without supervision. It was also reported that attending to a cardiac arrest soon after the course appeared to be key to reinforcing confidence. ${ }^{(17)}$ In lieu of actually encountering an IHCA, periodic team-based resuscitation drills should be organised to maintain skills and knowledge.

\section{CONCLUSION}

It is recommended that the LSCN should feature early in nurses' continuing education roadmap. The survey found that the majority of LSCN graduates and instructors felt that the course was useful and had enhanced their confidence to perform nurse-initiated resuscitation. To promote skill and knowledge retention, hospitals should organise frequent team-based resuscitation training. Nursing leadership can also assist in overcoming perceived barriers by publicising nurse-initiated resuscitation successes.

\section{REFERENCES}

1. American Heart Association. Highlights of the 2015 American Heart Association Guidelines Update for CPR and ECC [online]. Available at: https://eccguidelines. heart.org/wp-content/uploads/2015/10/2015-AHA-Guidelines-HighlightsEnglish.pdf. Accessed June 11, 2016

2. Nadkarni VM, Larkin GL, Peberdy MA, et al. First documented rhythm and clinical outcome from in-hospital cardiac arrest among children and adults. JAMA 2006; 295:50-7.

3. Ranse J. Cardiac arrest: can the in-hospital chain of survival be improved? Australas Emerg Nurs J 2006; 9:23-27.

4. Sandroni C, Nolan J, Cavallaro F, Antonelli M. In-hospital cardiac arrest: incidence, prognosis and possible measures to improve survival. Intensive Care Med 2007; 33:237-45.

5. Nolan JP, Soar J, Smith GB, et al. Incidence and outcome of in-hospital cardiac arrest in the United Kingdom National Cardiac Arrest Audit. Resuscitation 2014; 85:987-92.

6. Hillman K, Chen J, Cretikos M, et al. Introduction of the medical emergency team (MET) system: a cluster-randomised controlled trial. Lancet 2005; 365:2091-7.

7. Bellomo R, Goldsmith D, Uchino S, et al. A prospective before-and-after trial of a medical emergency team. Med J Aust 2003; 179:283-7.

8. Bellomo R, Goldsmith D, Uchino S, et al. Prospective controlled trial of effect of medical emergency team on postoperative morbidity and mortality rates. Crit Care Med 2004; 32:916-21.

9. Donnino MW, Salciccioli JD, Howell MD, et al. American Heart Association's Get With The Guidelines-Resuscitation Investigators. Time to administration of epinephrine and outcome after in-hospital cardiac arrest with non-shockable rhythms: retrospective analysis of large in-hospital data registry. BMJ 2014; 348:g3028.

10. Heng WJ, Seow E, Tham KY. Life support course for nurses in Singapore. Singapore Med J 2011; 52:557-9.

11. Roh YS, Issenberg SB, Chung HS. Ward Nurses' Resuscitation of Critical Patients - Current Training and Barriers. Eval Health Prof 2014; 37:335-48.

12. Heng KW, Fong MK, Wee FC, Anantharaman V. The role of nurses in the resuscitation of in-hospital cardiac arrests. Singapore Med J 2011; 52:611-5.

13. Bloom BS (ed), Engelhart MD, Furst EJ, Hill WH, Krathwohl DR. Taxonomy of Educational Objectives: The Classification of Educational Goals. Handbook I: Cognitive Domain. New York: David McKay Company, 1956.

14. Moser DK, Coleman S. Recommendations for improving cardiopulmonary resuscitation skills retention. Heart Lung 1992; 21:372-80.

15. Leith B. Retention of defibrillation training by intensive care nurses. Can Assoc Crit Care Nurs 1997; 8:9-11.

16. Hamilton R. Nurses' knowledge and skill retention following cardiopulmonary resuscitation training: a review of the literature. J Adv Nurs 2005; 51:288-97.

17. Murphy M, Fitzsimons D. Does attendance at an immediate life support course influence nurses' skill deployment during cardiac arrest? Resuscitation 2004; 62:49-54. 